\title{
Impact of Antimicrobial Stewardship Program on Vancomycin Usage: Costs and Outcomes at Hospital for Tropical Diseases in Ho Chi Minh City, Vietnam
}

\author{
Hai-Yen Nguyen-Thi' \\ Duy-Anh Nguyen (D) \\ Phuong-Thao Huynh ${ }^{2}$ \\ Nguyen Dang Tu Le (D) \\ 'Department of Pharmaceutical \\ Administration, University of Medicine \\ and Pharmacy at Ho Chi Minh City, Ho \\ Chi Minh City, Vietnam; ${ }^{2}$ Department of \\ Pharmacy, Hospital for Tropical Diseases, \\ Ho Chi Minh City, Vietnam
}

\begin{abstract}
Background: Nowadays, with the emergence of vancomycin-resistant strains, the clinical use of vancomycin has been followed closely by applying the antimicrobial stewardship program (ASP) to enhance effectiveness in treatment and reduce cost burden for patients.

Methods: A descriptive cross-sectional study at the Hospital for Tropical Diseases was conducted to assess the inpatient status assigned to intravenous vancomycin and factors associated with the cost of treatment during two periods of implementing ASP, which were i) from April 1, 2016 to March 31, 2018 (previous ASP-pASP) and ii) from June 1, 2018 to March 31, 2020 (new ASP-nASP).
\end{abstract}

Results: Among 1375 patients who met the sampling criteria, there were 601 and 774 patients in pASP and nASP, respectively. The rate of no improvement/mortality in the pASP was higher than that in nASP $(37.10 \%$ vs $25.98 \%, \mathrm{p}<0.05)$. The proportion of patients with two or more infection episodes in nASP is lower than that in pASP $(9.83 \%$ vs $18.64 \%, p<0.05)$. Besides, nASP has higher length of therapy (LOT) and higher day of therapy (DOT). The average treatment cost in the pASP is higher than that in the nASP, 1891.22 (95\% CI, 1713.462068.98) USD vs 1775.55 (95\% CI, 1576.22-1974.88) USD. There are seven factors $(p<0.05)$ that associate with the total cost of treatment (age, number of infection episodes, length of stay, discharge status, clinical department, LOT, DOT) in pASP. On the other hand, the nASP has five factors $(\mathrm{p}<0.001)$, in which the $\log (\mathrm{LOT})$ and age are not as statistically significant ( $\mathrm{p}=0.5127$ and 0.3852 , respectively) as in the pASP model.

Conclusion: The implementation and improvement of the ASP at the Hospital for Tropical Diseases have initially shown benefits for patients using intravenous vancomycin. Specifically, the ASP helps to reduce treatment costs, improve patient outcomes, reduce length of stay and decrease the average daily dose of vancomycin.

Keywords: antimicrobial stewardship program, vancomycin, Hospital for Tropical Diseases, Vietnam, treatment costs, outcomes

\section{Introduction}

The prevalence of gram-positive infection has continued to increase steadily over the past two decades and has become a common cause of community infection and a burden on the total cost of treatment. ${ }^{1,2}$ Among pathogenic gram-positive bacteria, Staphylococcus aureus, Streptococcus pneumoniae and enterococci stand out as the main cause of global antimicrobial resistance. ${ }^{3}$ These have led to an increasing use of vancomycin in clinical practice against gram-positive bacteria. ${ }^{4}$
Correspondence: Hai-Yen Nguyen-Thi Department of Pharmaceutical Administration, Faculty of Pharmacy, University of Medicine and Pharmacy at Ho Chi Minh City, 4I Dinh Tien Hoang Street, Ho Chi Minh City, Vietnam Tel +84938769626

Email haiyen@ump.edu.vn 
However, the emergence of vancomycin-intermediate and vancomycin-resistant Staphylococcus aureus and Enterococcus faecium (VRE) strains has resulted in a more rigorous approach to vancomycin restriction in use and to vancomycin treatment monitoring. 5,6 Applying antimicrobial stewardship programs (ASPs) in clinical practice improves the rationality of antibiotic use, clinical effectiveness and reduces cost of treatment. ${ }^{7}$ In Vietnam, the ASP started in 2016 according to Decision 772/QDBYT of the Ministry of Health; however, the implementation of hospitals still remained limited. ${ }^{8,9}$ Hospital for Tropical Diseases in Ho Chi Minh City (HTD) is one of the first hospitals to apply ASP, but the evidence of benefits and effectiveness of ASP has not been systematically assessed. In addition, the patients, who used vancomycin during treatment, should also be strictly monitored and the ASP strongly focuses on managing this group of antibiotics as well. For these reasons, this study was conducted to assess the inpatient status assigned to intravenous vancomycin and factors associated with the patient's cost of treatment during the implementation of ASP.

\section{Materials and Methods Study Design}

This study was conducted as a retrospective study at the HTD, between April 2016 and March 2020. HTD is a hospital specialized in infectious diseases with 500 beds (180 beds for children and 320 beds for adults), is also a terminal specialized hospital in the south. The study investigated the status of intravenous vancomycin treatment and the factors associated with the total treatment costs in two stages of applying ASP which were as follows: i) from April 1, 2016 to March 31, 2018 (previous ASP-pASP) and ii) from June 1, 2018 to March 31, 2020 (new ASP-nASP). During the nASP period, the hospital made some amendments and increased investment in management program and policy for antibiotic use. The summary of differences in policies between pASP and nASP is described in Table 1.

For vancomycin, at HTD, the recommended dose (for patients $>8$ years) is as follows: i) Loading dose (not compulsory): $25-30 \mathrm{mg} / \mathrm{kg}$ according to actual weight and ii) Maintenance dose: $15-20 \mathrm{mg} / \mathrm{kg}$ according to actual weight every $8-12$ hours. In case of patients with suspected risks of MRSA, vancomycin is indicated after a consultation between physicians and clinical pharmacists. However, at the pASP stage, most of the initial dose will maintain if the patient is clinically stable. In the nASP stage, treatment is monitored more closely and interventions are taken after receiving culture. These interventions include down the antibiotic scale according to antibiotic results after considering the clinical progression, considering the combination of antibiotics with the aim of expanding the spectrum of effects on pathogenic microorganisms, synergistically enhancing the bactericidal effect, reducing and preventing the emergence of resistance mutations during treatment.

\section{Data Collection}

\section{Evaluated Variables}

This study aims to evaluate the association between patient characteristics and clinical indicators with the total treatment cost of patients using intravenous vancomycin under administration of an antimicrobial stewardship program. The variables include i) baseline characteristics: age, gender, height, weight, hospital admission department and diagnosis of infection by ICD-10; ii) clinical outcome: discharge status, length of stay, infection episode, duration of vancomycin use and dose; iii) direct medical costs: drug costs (antibiotics, vancomycin, and non-antibiotics), bed cost and other costs.

\section{Inclusion and Exclusion Criteria}

Data were collected through retrospective full electronic medical records of inpatients. Inclusion criteria comprised i) inpatients who received intravenous vancomycin during treatment and ii) patients who had all needed information for the study (include baseline characteristics, drug indications, clinical outcome and direct medical costs). Exclusion criteria included i) inability to pay or get free medical bills; ii) patients $<18$ years of age; iii) on dialysis, peritoneal dialysis, and pregnant women (there is a large variation in pharmacokinetics in these patients); iv) patients with tuberculosis diagnosis (these patients were affected by another intervention that is not affected by the ASP).

\section{Data Analysis}

The costs in this study were estimated as the direct medical costs according to the payer's perspective (Vietnam Social Security) follow the sum-all cost and incidencebased approach. Infection episode is determined based on the date the antibiotic treatment was started. The next infection episode begins after at least 2 non-using antibiotic days after the previous infection episode or a relapse of 
Table I Summary Differences in Policies Between pASP and nASP

\begin{tabular}{|c|c|c|}
\hline & pASP & nASP \\
\hline How to stratify the patient & 3 groups & $\begin{array}{l}2 \text { groups, focusing on examining the risk of infection with multi- } \\
\text { resistant bacteria }\end{array}$ \\
\hline Number of antibiotics in $0 \mathrm{l}$ prescription & No specified & Maximum of 02 antibiotics \\
\hline Antibiotics need consultation & 10 antibiotics & All intravenous antibiotics and oral linezolid \\
\hline Return microbiological results & - & According to each step \\
\hline Blood transplant procedure & - & $\begin{array}{l}\text { Increased blood collection into } 2 \text { bottles, using fast bacteria } \\
\text { identifier Maldi-tof }\end{array}$ \\
\hline Limited antibiotic approval process & - & $\begin{array}{l}\text { Change the approval process, integrate into hospital management } \\
\text { software }\end{array}$ \\
\hline $\begin{array}{l}\text { Limited number of physicians who can approve } \\
\text { antibiotics }\end{array}$ & 2 physicians & 9 physicians \\
\hline $\begin{array}{l}\text { Limited number of clinical pharmacists who can approve } \\
\text { antibiotics }\end{array}$ & $\begin{array}{l}3 \text { clinical } \\
\text { pharmacists }\end{array}$ & 4 clinical pharmacists \\
\hline Compliance assessment, report results & $\begin{array}{l}\text { At the end of } \\
2017\end{array}$ & At the beginning of each month \\
\hline
\end{tabular}

a previous infection episode (for example, 1 st to 3 rd date of using antibiotics, counted as the first infection, 4th and 5th date of not using, and days 6th and 7th of using antibiotics again was counted as the second infection). Length of therapy (LOT) calculated for a course of infection is based on the number of days using antibiotics, regardless of the number of antibiotics used. Days of therapy (DOT) is similar to LOT; however, it does include the number of antibiotics used. ${ }^{10-12}$ The hospital classified the patient's discharge into six states as 1) recovery, 2) reduced, 3) hospital transfer, 4) unchanged, 5) severe and 6) mortality. In particular, to assess the outcome of patients, the study classified the outcome of patients into two main states: improvement (including recovery or reduced) and no improvement (lack of improvement in the clinical signs and symptoms or mortality, which include unchanged or severe or mortality or hospital transfer).

The study has been conducted over the years; therefore, all costs were converted into the analysis time (2020) by using the consumer price index (CPI), and then adjusted to US dollars, at the exchange rate updated as of March $2020(1 \mathrm{USD}=23,172.50 \mathrm{VND}){ }^{13}$

\section{Statistical Analysis}

Data were analyzed using $\mathrm{R}$ version 3.6.3. The study used a standard Q-Q plot probability chart to check the variables of LOT, DOT and treatment costs following the normal distributions. Continuous variables were expressed as the mean (SD [Standard Deviation]) for normal distribution or the median (interquartile range) for nonnormally distributed data. Categorical variables were expressed as number and percentages. The Chi-squared test is used to compare categorical variables, the $t$-test or Mann-Whitney test is used to compare continuous variables, and a p-value $<0.05$ was considered statistically significant. The study fitted the appropriate distribution of the cost data using Maximum Likelihood Estimate (MLE) and chose a distribution which had the minimum sum of squared error between the fits and the actual distribution ${ }^{14}$ The study analyzes factors associated with the patient's treatment costs using generalized linear regression model (Generalized Linear Model, GLM). GLM is one of the best options for data in medical research, which has high applicability and has been widely used around the world. ${ }^{15,16}$

\section{Ethical Statement}

Informed consent was obtained from patients or their caregivers by healthcare professionals during treatment at hospital in both pASP and nASP period. All patient information was anonymized and stored as unlinked data prior to analysis to prevent the disclosure of personal information. This study protocol was approved by Biomedical 
Research Ethics Council at the Hospital for Tropical Disease in HCMC under Decision 38/HDDD on September 30, 2019. This study was conducted in accordance with the Declaration of Helsinki.

\section{Results}

\section{Patient Characteristics}

Of the 1375 patients who formed the reference case, 601 patients were in the pASP group, 774 in the nASP groups. The number of male and female cases in the pASP period was $389(64.72 \%)$ and $212(35.28 \%)$, respectively, and in the nASP period 501 (64.73\%) and 273 (35.27\%), respectively. There is no statistically significant difference between the rate of men and women of the two periods $(p=0.1636)$. Inpatients using vancomycin at HTD in pASP and nASP stages have mean age of 44.3 and 43.4, respectively. Accordingly, in the pASP stage, the oldest case is 96 and 93 in nASP. The status of vancomycin usage was recognized in the study, which was widely distributed in 14/14 clinical departments, and was led by Department of B infection in both pASP and nASP stages. The patient characteristic results are shown in Table 2.

The results showed that the rate of no improvement/ mortality in pASP and nASP was $37.10 \%$ and $39.14 \%$, respectively $(p<0.05)$. Comparing the LOT and DOT data between the two periods in the study, the values in the pASP stage were both lower than that in the nASP stage (median 10 vs 12 and 14 vs 20, respectively). In the first stage, the control on prescribing restricted and broad-spectrum antibiotics was not really effective. Therefore, the use of these antibiotics shortens the treatment of infection, leading to a decrease in DOT and LOT. However, the downside is that the patient may experience undesirable effects of the drug and accelerate the antibiotic resistance. Therefore, in the nASP, the hospital is more concerned with the long-term impact of antibiotic use, as demonstrated by the promulgation of regulations on the number of prescribed antibiotics, on initial antibiotic selection and tighter control over limited antibiotics. Limiting the use of these antibiotics increases the duration of antibiotic use but has a beneficial long-term effect on resistance. Similarly, the control of vancomycin use in this study was consistent with the results of the study of general antibiotic use with the median of the vancomycin duration in the nASP phase (9 days) was longer than that in the pASP phase ( 8 days).

\section{Economic Burden}

The average total treatment cost of patients in the pASP is higher than that in the nASP, 1891.22 (95\% CI, 1713.462068.98) USD vs 1775.55 (95\% CI, 1576.22-1974.88) USD. During the nASP stage, the average of the drug costs and other costs decreased compared to the pASP stage. However, in the period of 2018 to 2020, the Ministry of Health issued circulars adjusting prices for medical examination and treatment services, leading to a higher mean hospital bed cost in the nASP period (277.02 USD) than in the pASP period (243.46 USD). The results of analyzing the treatment cost components of the cases in the two research periods are presented in Figure 1.

\section{Generalized Linear Model}

The study used a generalized linear model. The manual average cost, though, is numerically valuable. But in practical terms, the average self-imposed cost does not have much value. Hence, this figure can be viewed for reference with different regression models. The multicollinearity was not detected in the model. The multivariate linear regression model has the following form:

$$
\begin{aligned}
& \text { pASP stage* } \\
& \begin{aligned}
\log (\mathrm{TC}) & =0.1439 * \log (\text { Age })+0.1257 *(\mathrm{IE}) \\
& +0.7853 * \log (\mathrm{LOS})+0.1172 *(\mathrm{DS}) \\
& -0.1664 *(\mathrm{HAD})-0.6203 * \log (\mathrm{LOT}) \\
& +0.8138 * \log (\mathrm{DOT})+6.0809
\end{aligned}
\end{aligned}
$$

nASP stage*

$$
\begin{aligned}
& \log (\mathrm{TC})=0.1631 *(\mathrm{IE})+0.7409 * \log (\mathrm{LOS}) \\
& \quad+0.0631 * \log (\mathrm{S})+0.2229 *(\mathrm{DS}) \\
& \quad-0.2082 *(\mathrm{HAD})+0.3065 * \log (\mathrm{DOT}) \\
& \quad+6.1406
\end{aligned}
$$

*The coefficients have statistical significance with $p<0.001 . R^{2}$ is $58.73 \%$ and $63.68 \%$ for the pASP and nASP model, respectively.

To analyzing factors that correlate treatment costs of patients, multivariate generalized linear regression was applied (Table 3). Two models both have R-square above $50.00 \%(58.73 \%$ and $63.68 \%$ for pASP and nASP, respectively). The pASP model has seven statistically significant parameters $(\mathrm{p}<0.001)$. On the other hand, the nASP has six statistically significant parameters $(p<0.001)$, in which the $\log (\mathrm{LOT})$ is not as statistically significant as in the pASP model $(\mathrm{p}=0.5127)$. The days of hospitalization have the 
Table 2 Patient Characteristics

\begin{tabular}{|c|c|c|c|c|}
\hline & & pASP $(n=601)$ & $n A S P(n=774)$ & $p(* *)$ \\
\hline \multirow[t]{2}{*}{ Age } & Mean (SD) & $44.3(21.80)$ & $43.4(20.60)$ & 0.305 \\
\hline & Range & $18-96$ & $18-93$ & - \\
\hline \multirow[t]{2}{*}{ Gender, n (\%) } & Male & 389 (64.72\%) & $50 \mathrm{I}(64.73 \%)$ & $0.164^{(\mathrm{a})}$ \\
\hline & Female & $212(35.28 \%)$ & $273(35.27 \%)$ & \\
\hline \multirow[t]{3}{*}{ BMI } & Underweight $(<18.5)$ & $102(16.97 \%)$ & 134 (|7.3।\%) & $0.199^{(a)}$ \\
\hline & Normal (I8.5-24.9) & 419 (69.72\%) & 508 (65.63\%) & \\
\hline & Overweight (> 24.9) & $80(13.31 \%)$ & 132 (I7.05\%) & \\
\hline \multirow[t]{8}{*}{ Number of infection episode } & No. of episode & n (\%) & n (\%) & $0.243^{(\mathrm{a})}$ \\
\hline & I & 489 (81.36\%) & $698(90.17 \%)$ & \\
\hline & 2 & 81 (13.48\%) & $48(6.20 \%)$ & \\
\hline & 3 & 18 (2.99\%) & $18(2.33 \%)$ & \\
\hline & 4 & 08 (1.33\%) & $06(0.78 \%)$ & \\
\hline & 5 & $02(0.33 \%)$ & $03(0.39 \%)$ & \\
\hline & 6 & $02(0.33 \%)$ & $01(0.13 \%)$ & \\
\hline & 7 & $0 \mathrm{I}(0.18 \%)$ & - & \\
\hline \multirow[t]{2}{*}{$\mathrm{LOT}_{\text {vancomycin }}$} & Median (QI-Q3) & $8(3-11)$ & $9(4-12)$ & $<0.00 I^{(b)}$ \\
\hline & Range & $1-43$ & $\mathrm{I}-54$ & - \\
\hline Vancomycin dose $24 \mathrm{~h}$ & Mean (SD) & $2119(721.4 I)$ & $930(269.55)$ & $<0.001$ \\
\hline \multirow[t]{2}{*}{ LOT } & Median (QI-Q3) & $10(7-13)$ & $12(8-17)$ & $<0.00 \mathrm{I}^{(\mathrm{b})}$ \\
\hline & Range & $I-52$ & $\mathrm{I}-74$ & - \\
\hline \multirow[t]{2}{*}{ DOT } & Median (QI-Q3) & $14(10-22)$ & $20(12-29)$ & $<0.00 I^{(b)}$ \\
\hline & Range & $|-7|$ & $1-112$ & - \\
\hline \multirow[t]{6}{*}{ Discharge status $(*)$} & Recovery & 112 (I8.64\%) & $13 \mid(16.93 \%)$ & 0.093 \\
\hline & Reduced & 266 (44.26\%) & 340 (43.93\%) & 0.104 \\
\hline & Unchanged & 99 (16.47\%) & 56 (7.24\%) & 0.074 \\
\hline & Severe & 116 (19.30\%) & 139 (I7.96\%) & 0.092 \\
\hline & Mortality & $8(1.33 \%)$ & $6(0.78 \%)$ & 0.080 \\
\hline & Hospital transfer & - & 102 (13.16\%) & - \\
\hline \multirow[t]{9}{*}{ Infection characteristics } & Pneumonia & $40(6.66 \%)$ & $83(10.72 \%)$ & 0.072 \\
\hline & Meningitis & $62(10.32 \%)$ & $5 \mathrm{I}(6.59 \%)$ & 0.085 \\
\hline & Infective endocarditis & $7(1.16 \%)$ & $6(0.78 \%)$ & 0.080 \\
\hline & Septicemia & $27 \mathrm{I}(45.09 \%)$ & 285 (36.82\%) & 0.130 \\
\hline & Encephalitis, myelitis and encephalitis - spinal cord & $20(3.33 \%)$ & $18(2.33 \%)$ & 0.081 \\
\hline & Cellulitis & $38(6.32 \%)$ & 97 (I2.53\%) & 0.063 \\
\hline & Urinary tract infections & $8(1.33 \%)$ & $3(0.39 \%)$ & 0.080 \\
\hline & Skin and soft tissue infections & $6(0.99 \%)$ & $23(2.97 \%)$ & 0.078 \\
\hline & Others & 149 (24.80\%) & $208(26.87 \%)$ & 0.080 \\
\hline \multirow[t]{2}{*}{ LOS } & Median (QI-Q3) & $16(11-25)$ & $14(10-21)$ & $<0.00 I^{(b)}$ \\
\hline & Range & $3-300$ & $3-133$ & - \\
\hline
\end{tabular}

Notes: $(*)$ Statuses are classified according to the physician's clinical judgment recorded in the medical record. $\left({ }^{* *}\right)$ The $t$-test is used, unless stated otherwise, ${ }^{(a)}$ Chi-square test, ${ }^{(b)}$ Mann-Whitney.

Abbreviations: BMI, body mass index; LOT, length of stay; DOT, day of therapy.

greatest impact on the patient's treatment costs in both antibiotic management programs with the median at the stage of pASP and nASP was 16 and 14 days, respectively. The intercept coefficient in the total cost regression model of patients in pASP and nASP is 6.0809 and 6.1406, respectively. This parameter means that the median selfdetermined average cost of a patient was 51.99 USD in pASP stage and 59.65 USD in nASP stage. 

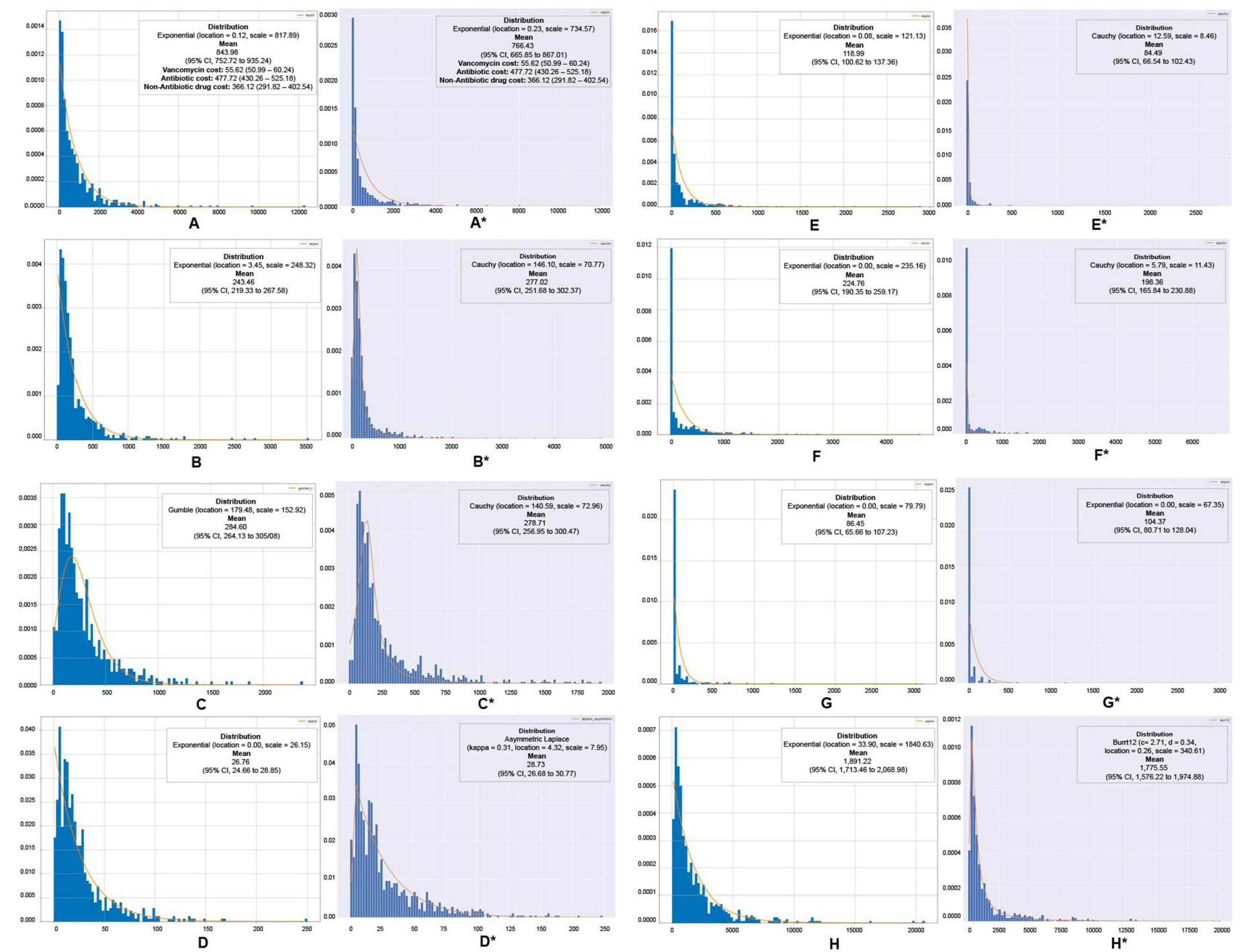

Figure I Mean treatment costs of patients and fitted distribution of cost data. (A, A*) Drugs cost; (B, B*) Bed cost; (C, C*) Testing cost; (D, D*) Image diagnosis cost; (E, E*) Medical supplies cost; (F, F*) Surgical procedures cost; (G, G*) Blood cost; (H, H*) Total cost. *The nASP period. Note: All costs will be converted to 2020 . Results are presented as Mean (95\% Cl) (USD).

The relationship between overall cost and LOS in hospital is described in Figure 2. The plot shows a nonlinear relationship between the total cost and the number of days hospitalized. Shaded areas represent raw data of cases, solid lines represent averages through data using limited cubic spline. Therefore, the spline function is used to estimate the patient's average total cost over the length of hospital stay. According to this model, cost increases most during the first 4 weeks in both pASP and nASP periods. After that, although costs have continued to increase, but not significantly compared to the beginning.

\section{Discussion}

In addition to the increasing interest in antibiotic resistance rates, current healthcare practices, especially in antibiotic using practice, have faced many challenges such as improving health outcomes, reducing medical errors and ensuring that treatment costs are affordable to patients. To address these challenges, the ASP has been introduced into clinical practice at HTD in 2016 and has been continually improved since then. The application of ASP initially showed positive results, such as reduction in costs, possible improvements in patient outcomes, reduction of the LOS as well as decrease in average daily dose of vancomycin. However, to be able to conclude the efficacy of vancomycin treatment, it is necessary to evaluate additional criteria including i) response status and clinical improvement after 24 to 48 hours of vancomycin use; ii) trough or AUC/MIC concentrations measured after drug administration; iii) adverse effects as well as risk of acute nephrotoxicity and iv) costs for monitoring, prevention and management of vancomycin-induced toxicity, as well as costs for staffs including doctors, pharmacists, clinical pharmacists and nurses. Even so, from the hospital's point 
Table 3 The Coefficients of Multivariate Generalized Linear Model for Cost Prediction

\begin{tabular}{|c|c|c|c|c|}
\hline \multirow[t]{2}{*}{ Parameter } & \multicolumn{2}{|l|}{ pASP } & \multicolumn{2}{|l|}{ nASP } \\
\hline & Coefficient (SE) & $p$ & Coefficient (SE) & $p$ \\
\hline Intercept & $6.0809(0.1715)$ & $<0.001$ & $6.1406(0.1498)$ & $<0.001$ \\
\hline $\log (\mathrm{Age})$ & $0.1439(0.0717)$ & 0.0453 & $0.0567(0.0652)$ & 0.3852 \\
\hline IE & $0.1257(0.0275)$ & $<0.001$ & $0.1631(0.0332)$ & $<0.001$ \\
\hline $\log (\mathrm{LOS})$ & $0.7853(0.0698)$ & $<0.001$ & $0.7409(0.0642)$ & $<0.001$ \\
\hline Sex & $0.0383(0.0248)$ & 0.1233 & $0.063 \mid(0.0222)$ & 0.0047 \\
\hline DS & $0.1172(0.0302)$ & $<0.001$ & $0.2229(0.027 \mathrm{I})$ & $<0.001$ \\
\hline HAD & $-0.1664(0.0154)$ & $<0.001$ & $-0.2082(0.0141)$ & $<0.001$ \\
\hline $\log (\mathrm{LOT})$ & $-0.6203(0.1182)$ & $<0.001$ & $0.0257(0.0393)$ & 0.5127 \\
\hline $\log (\mathrm{DOT})$ & $0.8138(0.0969)$ & $<0.001$ & $0.3065(0.0539)$ & $<0.001$ \\
\hline
\end{tabular}

Notes: TC (total cost), which is estimated from the model (the independent variable), is a continuous quantitative variable; IE (Number of infection episode): the number of infection episodes, is a discrete variable; LOS (length of stays) is a discrete variable; DS (discharge status): I if the state improve (recovery, reduced, hospital transfer), 2 if the state does not improve (unchanged, severe, mortality); $S$ (sex): I if male, 2 if female; HAD (hospital admission department): I if Intensive care-Resuscitation Antipoison Department, 2 if Pediatrics Department, 3 if Infectious Disease Department, 4 if Internal Medicine Department.

Abbreviation: SE, standard error.

of view, when implementing the ASP, these results were found to be effective. Therefore, we recommend supplementing/modifying the ASP program policy to evaluate the treatment benefits as well as the economics of vancomycin use.

Although it is a narrow-spectrum antibiotic, vancomycin was used in most clinical departments at the hospital. This condition can be explained by the empirical indication of vancomycin in a wide variety of infectious diseases such as pneumonia, meningitis, soft tissue skin infections, endocarditis, sepsis and other designation. The median length of vancomycin therapy (LOTvancomycin) increased from 8 (IQR, 3-11) to 9 (IQR, 4-12) days

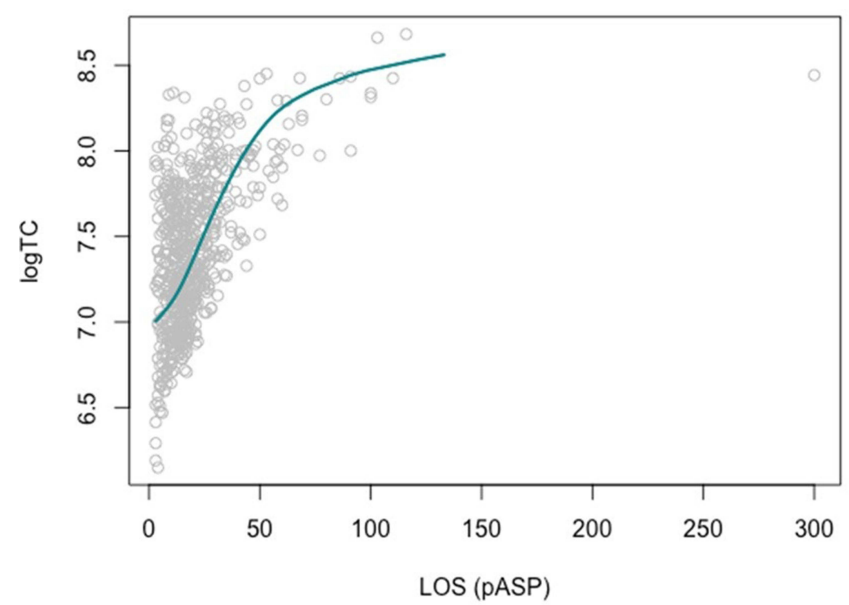

$(p<0.001)$. The duration of treatment in nASP stage was consistent with the recommended duration of vancomycin for patients who cannot tolerate penicillin or ceftriaxone therapy (4 weeks). ${ }^{18}$ In addition, the average vancomycin 24h dose was significantly reduced, from 2119 (721.41) to 930 (269.55) $\mathrm{mg}(\mathrm{p}<0.001)$. This will help reduce many clinical risks for the patients in the long term. ${ }^{19,20}$ In the pASP stage, when the introduction of an ASP was relatively new, the initial use of vancomycin was largely based on empiric dosing from physicians. On the other hand, when the patient is treated with the initial experienced dose level, if the clinical condition is stable or patient responds well to the drug, that dose level will continue to be maintained, from which the dose level is not used optimizingly for each patient which would results in rather high daily dosages. The daily dose level of the study in the pASP period was similar to that of the study by $\mathrm{Vu}$ et al, at Bach Mai hospital, which is the largest hospital in Northern Vietnam, the maintenance dose level using vancomycin was 2261 (1052) $\mathrm{mg}$ for ICU patients, and the maintenance dose level was $2000-3000 \mathrm{mg}$ for non-ICU patients with $\mathrm{CrCl}$ from $61-130 \mathrm{~mL} / \mathrm{min}^{26}$ However, in the nASP stage, changes and improvements to the ASP management protocol have resulted in a stricter control on the use of antibiotics in general and vancomycin in particular, and led to the dose adjustment of vancomycin based on disease characteristics of patients. During treatment, patients are monitored for drug response efficacy, and dose-adjustment interventions are made based on individual conditions to optimize the dose regimen. The dose regimen for the nASP stage is similar to that of Drayton A. Hammond's study with a mean dose level of 1148 (217) $\mathrm{mg}$ per day. ${ }^{27}$ The results showed that the use of

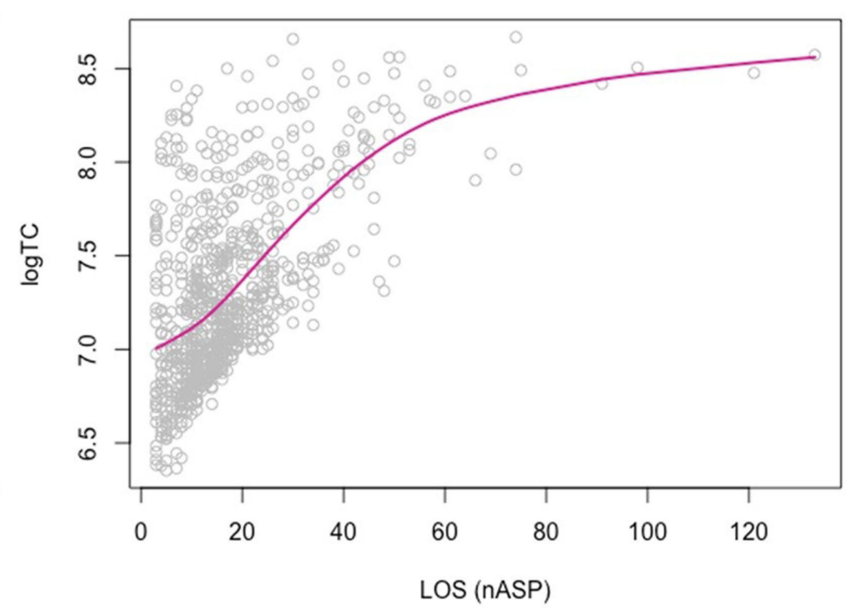

Figure 2 Total treatment cost (logTC) and length of stay (LOS) relationship of patients in the pASP and nASP period to the left and right hand side respectively. 
vancomycin in infectious diseases in this study was relatively consistent with the recommended indications for using vancomycin and the rate of using vancomycin with appropriate indications in pASP and nASP was $75.21 \%$ and $73.13 \%$, respectively. However, at the beginning of the administration of ASP, the vancomycin indications for infection have not been strictly controlled and vancomycin was used largely as empirical prescription of physician or as prescription to cover suspected bacteria. The factors, which associated with the inappropriate use of vancomycin, have been evaluated in several studies. ${ }^{21,22}$ However, ADR for continuing empirical inappropriate vancomycin treatment is less often studied. A study by Junior M.S., which analyzed the continuation of inadequate vancomycin therapy for 72 hours after initiation, showed that in more than $50 \%$ of cases, vancomycin use was continued due to severe clinical conditions, without documented records of gram-positive bacteria and with associated factors of being age under 60 , no hospitalization of Intensive Care Unit (ICU) and no leukopenia. ${ }^{22}$ Hospitals need to promote microbiological culture and clinical evaluation after negative culture results. Prolonged empirical treatment with no clear evidence of infection, treatment of negative cases similar to positive cases is common antibiotic abuse. $^{18}$

Predictive multivariate regression models of treatment costs are made by considering influencing factors including demographics and clinical outcomes. The model of treatment cost has relatively low R-square, in other words, some factors related to treatment cost have not been recognized. Despite this interest, as far as we know, no one has conducted a study to build a multivariate regression model to assess factors affecting treatment costs of vancomycin with inpatients. The R-square values in this study are still higher when comparing the R-square values in a similar study on the cost of treatment for Linezolid by MCKinnon et al (with R-square values in 2 population groups of $24 \%$ and $34 \%$ ). ${ }^{28}$ The LOS had the highest impact on the patient's total direct medical cost in the estimated models in both stages. The mean LOS in the nASP period was 17.44 (16.50-18.38, 95\% CI) days, which was significantly shorter than the 20.57 (19.05$22.11,95 \% \mathrm{CI})$ days in the prior period $(\mathrm{p}=0.048)$. This reduction was noted to be similar to a study in Hong Kong, in which the way of implementing the ASP is quite similar to implementation in the HTD, showed a decrease in LOS from 7.46 to 6.97 days $(\mathrm{p}<0.001){ }^{23}$ The LOS in this study was much shorter than the average hospital stays of 39.8 days reported in Junior M.S.'s study, a prospective survey conducted on all patients receiving vancomycin. ${ }^{22}$ However, it was quite similar to the study by Nicolas Perin in 2020 assessed the proportion of intensive care unit (ICU), which had an average hospital stay of about 23 days. $^{24}$

In terms of discharge status, compared with pASP period, the proportion of hospital discharge cases with cured or decreased status in nASP was lower $(60.85 \%$ in nASP vs $62.06 \%$ in pASP), especially in the last 3 months of nASP period. The explainable reason is that during the outbreak of the COVID-19 pandemic as well as during the Lunar New Year period, the hospital mainly kept treating inpatient patients who were severe with bad prognosis. Hence, the successful treatment probabilities of these cases were also be lower. In addition, the proportion of patients with two or more infection episodes in nASP stage is lower than the pASP stage $(9.83 \%$ vs $18.64 \%$, $\mathrm{p}<0.05)$. As for the results of number of infections in the nASP stage, the study found that the rate of infections was 01 episode higher than the pASP stage $(90.17 \%$ versus $81.36 \%$ ). In which, the rates of vancomycin use in the first infection episode in the pASP and nASP stages are 547 cases $(91.01 \%)$ and 734 cases $(94.83 \%)$; in the second infection are 46 cases $(7.66 \%)$ and 31 cases $(4.00 \%)$; in the third episode of infection were 07 cases $(1.16 \%)$ and 08 cases $(1.03 \%)$ and in the fourth episode of infection were 01 case $(0.17 \%)$ and 01 case $(0.13 \%)$, respectively. However, using retrospective data, the study can only determine the number of infections per patient during treatment but cannot classify whether the infection was a relapse from the previous episode or a new infection, as well as identifying the agent causing the new infection. In general, the rate of vancomycin use right at the first infection in both stages is high, over $90 \%$, and there is no use case after the 4 th infection. By identifying the role of ASP in the hospital to contribute to reducing inappropriate antibiotic use through 4 "Ds" including 1) Drugs: choosing the right drugs; 2) Dose, including route and frequency; 3 ) De-escalation to pathogen-directed therapy and 4) Duration: reducing drug dosing time. These results of the study showed that the antibiotic management policies of nASP may have helped the hospital to improve the effectiveness of infection's treatment as well as improve the infection control process. Therefore, it helps reduce the incidence of new infections after being admitted to the hospital in inpatient cases in the hospital. 
The drug costs account for a relatively high proportion, which was about $40 \%$ of total direct medical costs. Regarding the structure of drug costs, the proportion of drug costs of vancomycin accounted for about $6 \%$ and is similar in both pASP and nASP stages. The percentage of antibiotic costs at HTD accounted for $51.85 \%$ and $52.43 \%$ of the total drug cost in two stages, respectively. Compared to the results from the study conducted in 406 hospitals in the United States, most hospitals have antibiotic costs accounting for about $10-25 \%$ of the total drugs' cost, antibiotic costs at HTD are 2 to 5 times higher. ${ }^{25}$ This disparity can be explained by the fact that the HTD is the terminal specialist hospital for infectious diseases, so antibiotic is an important group of drugs, accounting for most of the drugs' expenditure. The increase in LOT value during the nASP period also contributes to the increase in the cost of the hospital bed. However, the increase in the cost of the hospital bed is largely due to the increment according to Inter-Circular No. 37/2015/TTLT-BYT-BTC. This regulatory bill took effect from March 1, 2016, with a gradually increment route from 2017 to $2019 .{ }^{17}$ The study estimated the fitted distributions of cost data to serve as the scientific basis for future Bayesian analysis and modeling studies for the ASP evaluation in hospital.

\section{Limitations}

There are several limitations in the study: i) outbreak of COVID-19 pandemic affecting hospital examination and treatment from January to March 2020; ii) research data were limited (because the hospital changed its hospital management system, it was difficult to re-access the previous database), so it was not possible to evaluate the nonimplementation of ASP; iii) for the retrospective data, the data cannot determine whether the following episode and the previous infection episode have the same causative agent or not.

\section{Conclusion}

The implementation and improvement of the ASP at the Hospital for Tropical Diseases have initially shown benefits for patients using vancomycin. Specifically, the ASP helps reduce the amount of vancomycin used, reduce hospital stay days and reduce treatment costs. There was also a change in factors related to treatment costs. However, the COVID-19 pandemic may compromise these results. Therefore, it is necessary to have further evaluations to serve as a basis to make appropriate policy for the hospital as well as to advise ASP policy of the city.

\section{Acknowledgments}

The authors would like to thank the board of directors and the hospital staffs of Hospital for Tropical Diseases for offering us a great opportunity to conduct our research at their sites.

\section{Funding}

There is no funding to report.

\section{Disclosure}

The authors report no conflicts of interest in this work.

\section{References}

1. Centers for Disease Control and Prevention. Monitoring hospitalacquired infections to promote patient safety-United States, 19901999. MMWR Morb Mortal Wkly Rep. 2000;49(8):149-153.

2. Di Pentima MC, Chan S. Impact of antimicrobial stewardship program on vancomycin use in a pediatric teaching hospital. Pediatr Infect Dis J. 2010;29(8):707-711. doi:10.1097/INF.0b013e318 $1 \mathrm{~d} 683 \mathrm{f} 8$

3. Woodford N, Livermore DM. Infections caused by Gram-positive bacteria: a review of the global challenge. $J$ Infect. 2009;59:S4S16. doi:10.1016/S0163-4453(09)60003-7

4. Pallares R, Dick R, Wenzel RP, Adams JR, Nettleman MD. Trends in antimicrobial utilization at a tertiary teaching hospital during a 15year period (1978-1992). Infect Control Hosp Epidemiol. 1993;14 (7):376-382. doi:10.1086/646765

5. Finks J, Wells E, Dyke TL, et al. Vancomycin-resistant Staphylococcus aureus, Michigan, USA, 2007. Emerg Infect Dis. 2009;15(6):943. doi:10.3201/eid1506.081312

6. Alliance for the Prudent Use of Antibiotics. Executive summary: select findings, conclusions, and policy recommendations. Clin Infect Dis. 2005;41(s4):S224-S227. doi:10.1086/430781

7. Dellit TH, Owens RC, McGowan JE, et al. Infectious Diseases Society of America and the Society for Healthcare Epidemiology of America guidelines for developing an institutional program to enhance antimicrobial stewardship. Clin Infect Dis. 2007;44 (2):159-177. doi:10.1086/510393

8. Thi-Hai-Yen Nguyen DV, Truong PT, Chi-Thuong Tang VC, et al. Implementation status of antimicrobial stewardship programs in hospitals: a quantitative analysis study in Ho Chi Minh city, Vietnam. Medpharmres. 2020;4(2):34-39. doi:10.32895/UMP.MPR.4.2.5

9. Ministry of Health. Decision No. 772/QD-BYT on the Issuance of the "Manual on Management of the Use of Antibiotics in Hospitals"; 2016.

10. Polk RE, Hohmann SF, Medvedev S, Ibrahim O. Benchmarking risk-adjusted adult antibacterial drug use in 70 US academic medical center hospitals. Clin Infect Dis. 2011;53(11):1100-1110. doi:10.1093/cid/cir672

11. Yarrington ME, Moehring RW. Basic, advanced, and novel metrics to guide antibiotic use assessments. Curr Treat Options Infect Dis. 2019;11(2):145-160. doi:10.1007/s40506-019-00188-3

12. Morris AM. Antimicrobial Stewardship Programs: appropriate Measures and Metrics to Study their Impact. Curr Treat Options Infect Dis. 2014;6(2):101-112. doi:10.1007/s40506-014-0015-3

13. The Global Economy. Vietnam:Consumer Price Index (CPI). 2020

14. Karian ZA, Dudewicz EJ. Handbook of Fitting Statistical Distributions with R. CRC Press; 2016. 
15. Manning WG, Mullahy J. Estimating log models: to transform or not to transform? J Health Econ. 2001;20(4):461-494. doi:10.1016/ S0167-6296(01)00086-8

16. Pagano E, Petrelli A, Picariello R, Merletti F, Gnavi R, Bruno G. Is the choice of the statistical model relevant in the cost estimation of patients with chronic diseases? An empirical approach by the Piedmont Diabetes Registry. BMC Health Serv Res. 2015;15(1):1-7. doi:10.1186/s12913-015-1241-1

17. Ministry of Health, Ministry of Finance. Joint Circular No. 37/2015/ TTLT-BYT-BTC "Unifying Prices for Medical Examination and Treatment Services Covered by Medical Insurance among Hospitals of the Same Level across the Country"; 2015.

18. Leekha S, Terrell CL, Edson RS. General principles of antimicrobial therapy. Mayo Clin Proc. 2011;86(2):156-167. doi:10.4065/ mcp.2010.0639

19. Rybak M, Lomaestro B, Rotschafer JC, et al. Therapeutic monitoring of vancomycin in adult patients: a consensus review of the American Society of Health-System Pharmacists, the Infectious Diseases Society of America, and the Society of Infectious Diseases Pharmacists. Am J Health Pharm. 2009;66(1):82-98. doi:10.2146/ ajhp080434

20. Aljefri DM, Avedissian SN, Rhodes NJ, Postelnick MJ, Nguyen K, Scheetz MH. Vancomycin area under the curve and acute kidney injury: a meta-analysis. Clin Infect Dis. 2019;69(11):1881-1887. doi:10.1093/cid/ciz051

21. Roustit M, François P, Sellier E, et al. Evaluation of glycopeptide prescription and therapeutic drug monitoring at a university hospital. Scand J Infect Dis. 2010;42(3):177-184. doi:10.3109/00365540 903413614
22. Junior MS, Correa L, Marra AR, Camargo LFA, Pereira CAP. Analysis of vancomycin use and associated risk factors in a university teaching hospital: a prospective cohort study. $B M C$ Infect Dis. 2007;7(1):1-6. doi:10.1186/1471-2334-7-88

23. Ng CK, Wu TC, Chan WM, et al. Clinical and economic impact of an antibiotics stewardship programme in a regional hospital in Hong Kong. Qual Saf Health Care. 2008;17(5):387-392. doi:10.1136/ qshc. 2007.023267

24. Perin N, Roger C, Marin G, et al. Vancomycin serum concentration after $48 \mathrm{~h}$ of administration: a 3-years survey in an intensive care unit. Antibiotics. 2020;9(11):793. doi:10.3390/antibiotics9110793

25. Doron S, Nadkarni L, Price LL, et al. A nationwide survey of antimicrobial stewardship practices. Clin Ther. 2013;35(6):758-765. doi:10.1016/j.clinthera.2013.05.013

26. Vu DH, Nguyen DA, Delattre IK, et al. Determination of optimal loading and maintenance doses for continuous infusion of vancomycin in critically ill patients: population pharmacokinetic modelling and simulations for improved dosing schemes. Int $J$ Antimicrob Agents. 2019;54:702-708. doi:10.1016/j.ijantimicag.2019.09.018

27. Hammond DA, Atkinson LN, James TB, Painter JT, Lusardi K. Effects of staff education and standardizing dosing and collection times on vancomycin trough appropriateness in ward patients. Pharm Pract (Granada). 2017;15(2):949. doi:10.18549/PharmPract.2017.02.949

28. MCKinnon PS, Sorensen SV, Liu LZ, Itani KM. Pharmacoeconomics impact of linezolid on economic outcomes and determinants of cost in a Clinical Trial Evaluating patients with MRSA complicated skin and soft-tissue infections. Ann Pharmacother. 2006;40 (6):1017-1023. doi:10.1345/aph.1G728
Risk Management and Healthcare Policy

\section{Publish your work in this journal}

Risk Management and Healthcare Policy is an international, peerreviewed, open access journal focusing on all aspects of public health, policy, and preventative measures to promote good health and improve morbidity and mortality in the population. The journal welcomes submitted papers covering original research, basic science, clinical \& epidemiological studies, reviews and evaluations,

\section{Dovepress}

guidelines, expert opinion and commentary, case reports and extended reports. The manuscript management system is completely online and includes a very quick and fair peer-review system, which is all easy to use. Visit http://www.dovepress.com/testimonials.php to read real quotes from published authors. 